\title{
¿Qué falta por comprender sobre el concepto autoevaluación (del aprendizaje) en educación superior? Una mirada diferente desde su historia ${ }^{1}$
}

\author{
O que falta entender sobre o conceito de autoavaliação \\ (da aprendizagem) no ensino superior? Um olhar diferente de sua história \\ What is missing to comprehend about the concept self-assessment \\ (of learning) in higher education? A different view from its history
}

Miglena Kambourova ${ }^{1}$ - ${ }^{1}$ Universidad de Antioquia | Facultad de Medicina | Departamento de Educación Médica | Medellin | Antioquia | Colombia. Contato: miglena.kambourova@udea.edu.co. ORCID: https://orcid.org/0000-0001-9194-3132

Resumen: Si bien el concepto autoevaluación del aprendizaje ha sido desarrollado e investigado por diferentes autores en las últimas décadas, parece que aún genera desconfianza y dudas entre los profesores, lo cual se traduce en su poca aplicación en el proceso de enseñanza-aprendizaje de la educación superior. Por ello, en este artículo se propone realizar una aproximación a su historia desde el enfoque hermenéutico - lo cual implica analizar la evolución desde la palabra, su conversión a término y, posteriormente, a concepto - y desde lo didáctico para develar significados que han quedado pendientes a la comprensión del concepto en su totalidad (lo psicológico-sociológico, lo filosófico-ontológico y lo pedagógicodidáctico), con el fin de generar reflexiones en torno al tema, proponer otra visión frente a la autoevaluación y abrir un espacio a futuras discusiones.

Palabras clave: Autoevaluación del aprendizaje. Educación superior. Historia del concepto autoevaluación.

Resumo: Embora o conceito de autoavaliação da aprendizagem tenha sido desenvolvido e investigado por diversos autores nas últimas décadas, parece que ainda gera desconfiança e dúvidas entre os professores, o que se traduz em sua pequeño índice de aplicação no processo de ensino-aprendizagem do ensino superior. Por isso, este artigo se propõe a fazer uma abordagem de sua história a partir da hermenêutica - o que implica analisar a evolução da palavra, sua conversão ao termo e, posteriormente, ao conceito - e da didática para revelar significados cuja compreensão de seu conceito ficou pendente (o psicológico-sociológico, o filosófico-ontológico e o pedagógico-didático), a fim de gerar reflexões sobre o assunto, propor outra visão contra a autoavaliação e abrir um espaço para futuras discussões.

Palavras-chave: Autoavaliação da aprendizagem. Educação superior. História do conceito de autoavaliação.

Abstract: Although the concept self-assessment of learning has been developed and investigated by different authors during the last decades, it seems that it still generates distrust and doubts among teachers, which has resulted in its little application in the teaching-learning process of higher education. This article suggests an approximation to its history from the hermeneutical approach - which implies analyzing the evolution from the word, its conversion to term and, later, to concept - and from the teaching (didactics) approach to reveal meanings that are necessary for the comprehension of the concept in its totality (the psychological-sociological, the philosophical-ontological and the pedagogical-didactic). The purposes of this article are to generate reflections on the topic, to propose another vision toward self-assessment and to open the possibility for future discussions.

Keywords: Self-assessment of learning. Higher education. History of the concept self-assessment.

- Recebido em: 5 de junho de 2019 • Aprovado em: 6 de julho de 2020

DOI: http://dx.doi.org/10.1590/S1414-40772020000300007

Este é um artigo publicado em acesso aberto sob uma licença Creative Commons

https://creativecommons.org/licenses/by-nc/4.0/

\footnotetext{
${ }^{1}$ Parte de este artículo (lo referido a la historia del concepto autoevaluación) es tomado de la tesis doctoral $L a$ autoevaluación como componente del sistema didáctico: sus fines formativos en la educación superior, financiada por Colciencias (convocatoria 727 de 2015).
} 


\section{Introducción}

Si bien la autoevaluación ha sido un tema de interés en diferentes esferas de la vida humana durante décadas, tomó mucha importancia en la educación superior a partir de la década de 1990, principalmente por dos razones. Por un lado, la preocupación por garantizar la calidad de la educación impulsó la instauración de organismos gubernamentales con potestad de acreditar instituciones educativas y sus programas. Así, por ejemplo, en Colombia se creó el Sistema Nacional de Acreditación, con la Ley 30 de 1992 (MINISTERIO DE EDUCACIÓN NACIONAL, 2007), y en Brasil, el Sistema Nacional de Evaluación de la Educación Superior, por medio de Ley 10.861 de 2004 (JESÚS; BEDRITICHUK, 2018). En dichos procesos de acreditación, la autoevaluación es un elemento clave, pues proporciona una visión interna que no siempre se percibe desde el exterior: "representa no sólo una ventana para el autoconocimiento, sino también la posibilidad de que la propia institución revise y actualice sus metas" (JESÚS; BEDRITICHUK, 2018, p. 630).

En segundo lugar, la necesidad de armonizar distintos sistemas educativos y de modernizar la enseñanza tradicional, al generar mayor flexibilidad y movilidad, llevó a la creación del Espacio Europeo de Educación Superior (EEES), que se formalizó con la firma de la Declaración de Bolonia (1999). En el marco de las reformas, para muchas universidades, tanto dentro como fuera del contexto europeo, la innovación en la evaluación se convirtió en una apuesta esencial que ha permitido promover la autoevaluación del aprendizaje con los estudiantes.

Pese a ello, su aplicación, junto con la de otros métodos evaluativos diferentes a los tradicionales, aún es mínima, como lo confirma el estudio de Tronchin, Pedro y Rezende (2017, p. 769). Los autores concluyen que si bien los profesores también aplican tipos de evaluación “considerados más participativos y consonantes a una propuesta curricular globalizante", como la autoevaluación, estos se realizan, por ejemplo: "de manera incipiente en las disciplinas impartidas por la EEUSP [Escuela de Enfermería de la Universidad de São Paulo] y en las disciplinas optativas, en menor proporción”. En este mismo sentido, Álvarez Rojo et al. (2011), y Cabrales Salazar (2010), en el contexto español y colombiano respectivamente, encontraron que la evaluación en la universidad es aún tradicional, y no incluye o considera muy poco la autoevaluación del aprendizaje.

Entonces, la preocupación por garantizar la calidad de la educación superior con base en una enseñanza que corresponda a la actualidad, en busca de una respuesta a las necesidades 
del contexto histórico de nuestra época, ha promovido la autoevaluación como una de las posibles soluciones, tanto en el ámbito institucional como personal.

Sin embargo, llama la atención que si bien las instituciones educativas de nivel superior deben aplicar la autoevaluación como requisito obligatorio para recibir su acreditación y, por tanto, su reconocimiento social, la autoevaluación del aprendizaje realizada por el estudiante y promovida por el profesor no logre posicionarse en el aula universitaria como debería, pues aún es esporádica y aislada. ¿Por qué sucede ello? ¿Existen significados de la autoevaluación que aún no se hayan descubierto o cuya comprensión haya quedado pendiente? ¿Hay herencias que frenan su abordaje en el tiempo actual?

Con la intención de reflexionar sobre estas inquietudes y ofrecer algunas ideas al respecto, este artículo presenta una aproximación a la historia del concepto autoevaluación desde el enfoque hermenéutico, por medio del proceso PRACCIS (prejuicios, reflexión, análisis, comparación, comprensión, interpretación y síntesis), el cual consiste en indagar por la etimología de la palabra y detectar cuándo esta se convierte en "término al interior del lenguaje científico hasta que se instaura como concepto, cuando es usado por una escuela de pensamiento específico" (GONZÁLEZ AGUDELO, 2011, p. 137).

Pero no solamente se apuesta por la comprensión del concepto autoevaluación desde la hermenéutica, sino desde la didáctica, particularmente la universitaria, pues en él se develan significados que generan reflexiones sobre el rol del profesor en la formación de personas, profesionales y ciudadanos. Al comprender la autoevaluación del aprendizaje desde dicha perspectiva se podría dar el paso hacia su enseñanza planeada, sistemática e intencionada.

\section{Aproximación a la historia del concepto autoevaluación}

La palabra autoevaluación está compuesta por dos partes. Si bien existen dos versiones sobre el origen de la raíz “evaluación” -el verbo "valer”, del latín valere, con el significado "ser fuerte, vigoroso, potente" (COROMINAS, 1987, p. 596), y “valuar”, del francés évaluer como "valorar"-, en ambas se percibe la idea de una cualidad, entendida como "elemento o carácter distintivo de la naturaleza de alguien o algo" (REAL ACADEMIA ESPAÑOLA, 2001) que la hace digna de tener valor. Pero, ¿qué significa valor?

El concepto valor se refiere a lo económico y lo filosófico. En el Diccionario de ética y de filosofía moral (CANTO-SPERBER, 2001, p. 1173) se menciona que "desde la Antigüedad la palabra ha sido usada para indicar la utilidad o el precio de los bienes materiales y la dignidad o el mérito de las personas [...]". El significado económico del término inicialmente fue de uso 
amplio y se convirtió en fundamento de la ciencia económica. Aproximadamente a finales del siglo XIX y principios del XX, el término valor se extendió desde su significado inicial hacia la filosofía.

El filósofo español Ortega y Gasset (1883-1955) afirma que los valores son “cualidades de las cosas" (2004, p. 29), las cuales pueden ser propias, es decir, aquellas que "las cosas poseen por sí mismas, independientemente de su relación con otras cosas" (p. 30); y relativas, que no son visibles a "los ojos de la cara", son "faena del intelecto" (p. 31). Aunque el autor admite que los valores son en general objetivos, también reconoce que son subjetivos, pues la estimación es una "función psíquica real — como el ver, como el entender- en que los valores se nos hacen patentes. Y viceversa, los valores no existen sino para sujetos dotados de la facultad estimativa, del mismo modo que la igualdad y la diferencia sólo existen para seres capaces de comparar" (p. 32).

Entonces, desde sus raíces, la palabra evaluar y su derivado "evaluación” se refieren a aspectos y características de un objeto y/o sujeto cuyo valor es reconocido por alguien (pues valorar es un acto meramente humano). Ello se logra al comparar dichas cualidades a partir de un punto de referencia, con propiedades definidas por medio de un juicio; por ejemplo, al diferenciar a una persona sana de una enferma, una piedra valiosa de una corriente, un trabajo excelente de uno mediocre.

Estos mismos significados de la evaluación están presentes en la autoevaluación; no obstante, el contraste evidente se encuentra en el prefijo "auto", proveniente del griego " $\alpha v \tau o$ ", que significa "uno mismo". La autoevaluación es dar valor a sí mismo, ser su propio evaluador, al estimar su proceso y sus cualidades de aprendiz.

La valoración del conocimiento aprendido -sin denominarse evaluación o autoevaluación- desempeñó un papel esencial en la educación de la Antigua Grecia, concretamente en la enseñanza del filósofo Sócrates (470a.C.-399a.C), quien utilizó diálogos aplicando la ironía y la mayéutica (el llamado método socrático). Las preguntas y las respuestas intencionadas se constituían en una provocación intelectual para incitar el pensamiento, pues, mediante sus diálogos, Sócrates conducía "a la consciencia de sí, que también es consciencia del otro, pues comprender qué somos implica conocer la naturaleza propia del ser humano" (MACARIO OCAMPOS, 2017, p. 48).

La cultura griega y los dos principios "cuidarse de sí mismo" y "conócete a ti mismo" son referenciados por Foucault (1991, p. 50) en su obra Tecnologías del yo. Desde la perspectiva de Lanz (2012, p. 40-46), como prácticas de la subjetividad: 
[...] el cuidado de sí es un problema vinculado a las prácticas pedagógicas que, en sentido general, tiene que ver con la formación no para aprender algo exterior, un cuerpo de conocimientos, sino una educación para propiciar el ejercicio de la reflexión del educando con respecto a sí mismo, con respecto a la experiencia que uno tiene de sí mismo [...] El cuidado de sí, que puede traducirse como formación de sí, como conocimiento de sí, como práctica de sí, resulta ser una hermenéutica del sujeto mediante la cual se busca que éste se escuche y se mire a sí mismo, se interprete como persona, para que pueda formar-se y así pueda soportar como corresponde todos los acontecimientos posibles, todas las aflicciones y problemas que puedan afectarlo durante toda la vida [...] El cuidado de sí [...] expresa una actitud consigo mismo, pero también con lo otro, con los otros y con el mundo [...]. Si entrelazamos el concepto de cuidado de sí, señalado por Foucault, con el de Bildung, propuesto por Gadamer, la formación [...] corresponde más con la autoformación [la cual] surge más del proceso interior, se encuentra por ello ligado al sentido de lo ético y estético, no busca sólo un conocimiento profesional sino fundamentalmente bueno por lo cual se encuentra en permanente relación con la subjetividad [...] El papel del maestro es hacer que cada alumno llegue a hacerse a sí mismo [...] [Nietzsche se refiere a llegar a ser lo que se es. ¿Cómo se logra?] Esta apuesta reclama dos cosas del maestro: a) tiene que dejar pensar a los alumnos por sí mismos, esto es, permitirles tener confianza en sí mismos. b) quien educa no puede pretender que lo imiten, no puede pretender convertir a los educandos en fieles discípulos a imagen y semejanza de su yo. Quien educa debe abrir los caminos para que el yo y el tú se comuniquen para compartir lo que son y para enriquecerse mutuamente.

$\mathrm{Al}$ analizar estas ideas se comprende que entre más uno conozca sobre su interior, sobre su particularidad, más fácilmente podrá encontrar el camino correcto para lograr su proyecto de vida y potenciar sus capacidades en busca de su libertad, felicidad y sentido de la vida. Por lo tanto, el maestro debe creer plenamente en quien aprende, en su potencial inacabable, para así estimularlo, motivarlo, entusiasmarlo y ayudarlo a construir aprendizaje; a adquirir competencias, valores, actitudes, y a superar sus dificultades académicas y personales; a ser él mismo, único e irrepetible. Pues la formación, entendida desde el bildung, es "el auto-cultivo interior que depende del exterior, pero que es mucho más noble que el mundo exterior y apunta a una realización armónica de la totalidad interna" (HORLACHER, 2014, p. 40).

En los siglos XI y XII, en la Edad Media, cuando nacieron las primeras universidades, la autoevaluación aún no figuraba como palabra y tampoco se encontraron indicios para considerar que en la enseñanza se aplicaban métodos para estimular la reflexión personal y lograr la autoevaluación de conocimientos por parte de los estudiantes.

Desde finales del siglo XIX, ya en la Edad Moderna, se crearon las condiciones para el surgimiento del término autoevaluación. Así, cuando la psicología logró separarse de la filosofía y declararse ciencia, entre la producción científica se destacó el libro The Principles of Psychology (Principios de psicología) (1890) del filósofo estadounidense William James (1842-1910). Su capítulo 10, denominado "Consciousness of self” ("Consciencia de sí mismo"): "marcó la introducción del 'self' como un determinante principal del pensamiento 
humano, el sentimiento y el comportamiento, y como susceptible de ser comprendido por los procedimientos de investigación empírica”, según Ashmore y Jussim (1997, p. 3).

A partir de este momento, los conceptos "self and identity" comenzaron a utilizarse con mucha frecuencia en diferentes esferas: en los medios de comunicación, en las ciencias sociales, en la psicología; lo cual se reflejó en el aumento de producciones sobre ellos. Los trabajos desde la psicología en particular eran una clara muestra del intento de esa ciencia por profundizar en lo íntimo del ser humano, para comprender su mente, su consciencia, su capacidad de pensarse y de conocerse, lo cual no tardó en manifestarse en el desarrollo de nuevos conceptos.

Entre 1930 y 1940, cuando se estima que nació el término evaluación, algunos psicólogos centraron su interés en el self, y desarrollaron medidas de autorreportes de la autoestima; estos consistían básicamente en la evaluación de la actitud hacia uno mismo, probablemente en concordancia con la proliferación de los test, muy de moda en la época.

Tal vez, la combinación de los avances en el self y la evaluación permitieron el surgimiento del término autoevaluación en la década de 1930, hecho que podría ser confirmado por "la existencia de registros de algunas investigaciones sobre autoevaluación (DIGGOR, 1966; SUMNER, 1932) en relación con el aprendizaje de estudiantes universitarios en este periodo", según Lasonen (1995, p. 202). De igual manera lo expresa Brew (1995), quien aclara que muchas de ellas fueron reportadas en Estados Unidos.

Se considera que la educación progresista relacionada con John Dewey (1859-1952) o la escuela nueva en general, como movimientos pedagógicos, eran la base del pensamiento educativo humanista que surgió desde la década de 1950, lo que por su lado impulsó el desarrollo de teorías sobre el estudiante como aprendiz autodirigido.

A partir de la década de 1960 emergieron estudios más sistemáticos sobre la autoevaluación que primordialmente investigaban la coincidencia de las calificaciones de autoevaluación de estudiantes en comparación con las calificaciones de sus profesores. Al parecer, la autoevaluación como término ya tomó más fuerza en el medio científico en dicha época, dado que "entre las publicaciones del periodo se mencionaban diferentes títulos con la palabra 'self' como la autoevaluación, el auto-concepto, la auto-percepción y la autoestimulación" (ASHMORE; JUSSIM, 1997, p. 4).

El término autoevaluación se nutrió con significados desde la psicología humanista, la cual se gestó durante algunos años, pero de la que oficialmente solo se empezó a hablar entre 1961 y 1962. "La psicología de la Tercera Fuerza, como algunos la llaman, es en su mayor parte una reacción a las grandes insuficiencias de las psicologías conductuales y freudianas en su tratamiento de la naturaleza superior del hombre" (MASLOW, 1968, p. 686). Fue impulsada 
por pensadores progresistas y psicólogos; Carl Rogers (1902-1987) y Abraham Maslow (19081970) están entre los más destacados. A la nueva psicología le interesaban la persona como un todo, "sin fragmentaciones como la conducta o el inconsciente", y "los fenómenos más positivos y sanos del ser humano como [...] la autenticidad y el arte de ser uno mismo, porque de allí debía provenir el sentido de respeto y responsabilidad" (RIVEROS AEDO, 2014, p. 138).

El psicólogo estadounidense Carl Rogers a lo largo de su vida desarrolló ideas en torno a la personalidad y la educación centrada en la persona. Muchos de los elementos que llegaron al campo semántico de la autoevaluación - este "sí mismo" que se valora - se nutrieron de sus teorías, según las cuales hay una esfera de experiencia propia de cada individuo y dentro de ella se encuentra el self, una entidad flexible y móvil, un proceso y cambio constante; por lo tanto, los individuos son capaces de conseguir el crecimiento y el desarrollo personal. Existe el self ideal, "el concepto de sí mismo que la persona anhela y al cual le asigna un valor superior" (ROGERS, 1959, p. 200), que se opone al self real.

Para Frager y Fadiman (2001, p. 428), cuando recibimos realimentación de otros e interactuamos con ellos tenemos oportunidades para "conocernos mejor. Las relaciones constituyen las mejores ocasiones para desenvolvemos de manera plena, en armonía con el self, el prójimo y el entorno". Entonces, ¿el profesor, desde lo didáctico, puede potenciar el desarrollo del estudiante como persona -profesional y ciudadano-, por medio de la autoevaluación? O acaso ello representa una contradicción; es decir, si la autoevaluación se comprende como un asunto tan personal, ¿el otro puede intervenir en ella?

Es tan significativa la existencia del otro para el self que esta relación también fue objeto de estudio de los sociólogos; por ejemplo, los aportes de Charles Cooley (1864-1929) referidos al "yo reflejo", también llamado "yo social", mencionan que "el concepto que se tiene de sí mismo depende directamente de las opiniones y acciones de otros" (WEBSTER; SOBIESZEK, 1978, p. 41), las cuales pueden ser correctas o incorrectas.

En cuanto a la educación centrada en la persona, Rogers consideraba que era necesario hacer de cada persona el evaluador y el guía de su propia experiencia; pensaba que dirigirse al intelecto e ignorar los sentimientos, desconfiar del estudiante, es un error, pues "los sentimientos forman parte de la persona entera y que educar sólo la mente es una limitación innecesaria" (CLONINGER, 2003, p. 430). Pero desde la evaluación del profesor, ¿cómo se considera al estudiante?, ¿es un todo o es fraccionado en partes: cognición y emoción?

La persona misma, el self, puede valorar su aprendizaje mejor que nadie, por ser la única que realmente sabe lo que ha aprendido; cuánto le ha afectado en su ser integrar lo cognitivo, 
lo emocional y lo social. Por lo tanto, la autoevaluación es un proceso íntimo, muy personal, propio, legítimo, necesario y válido. Pero no por ello, por ser subjetivo, se debe despreciar o ignorar en el proceso formativo de la educación superior. Al contrario, hay que apoyarse con más fuerza en este ser que está en la búsqueda constante del conocimiento y la comprensión de sí mismo. Entonces, ¿por qué en las universidades no se utiliza la autoevaluación del estudiante de la misma manera como se aplica la evaluación del profesor?

La década de 1970 permitió agregar otros significados a la autoevaluación. Al final de esta época, en el Reino Unido, y en menor medida en Australia, Noruega y Suecia, según Gairín Sallán (1997), se iniciaron procesos de reformas curriculares en los que cobró importancia la autoevaluación. En este mismo sentido, Taras (2015, p. 3) relata que "los discursos sobre la autonomía e independencia del estudiante en los años 70 y 80 crearon una ruptura entre tutores y estudiantes", lo que impulsó ideas alrededor de las capacidades de los estudiantes de trabajar sin el apoyo directo e inmediato de los profesores.

En esta época la autoevaluación se conectó con las profesiones, con lo laboral, con el conocimiento de contenidos; en definitiva, se "asoció con la actualización ocupacional y el aprendizaje experiencial" (HERON, 1988, p. 203, como se cita en LASONEN, 1995, p. 202). Además, se consideró y comprendió "la función educativa de la autoevaluación de los estudiantes" (BOUD, 1991, como se cita en LASONEN, 1995, p. 202), lo que podría significar que a partir de entonces se habla del concepto autoevaluación.

Así las cosas, la autoevaluación se abría camino y poco a poco comenzaba a ser involucrada en la vida universitaria, primordialmente en forma de autoevaluación sumativa (con calificación), y concretamente, "cuando la autoevaluación se menciona se refiere al modelo estándar" (TARAS, 2015, p. 4). Dicho modelo requería de los "estudiantes evaluar su propio trabajo de acuerdo con criterios y estándares acordados" (p. 3). Los estudiantes "utilizan criterios para juzgar, proporcionar comentarios [se incluían anotaciones sobre las fortalezas y las debilidades percibidas que permitían hacer el juicio] y calificar su trabajo antes de enviarlo a los tutores" (TARAS, 2010, p. 203).

Heron (1988, Parte 5, párrafo 2) señala que la autoevaluación como proceso de reflexión sobre las etapas del propio aprendizaje es una condición necesaria para lograr la autodirección, pues "evaluar cómo aprendo y cómo proporciono evidencia de lo que he aprendido es realmente más fundamental que evaluar lo que he aprendido [...]". Se trata, entonces, de tener capacidades como la observación, el análisis, la comparación, entre otras, para comprender el camino que se recorrió hasta obtener un determinado resultado, pues al tener consciencia de las etapas pasadas es posible volver a seguir los pasos y producir cosas nuevas. 
Desde la década de 1990, la autoevaluación ha sido mucho más abordada por diferentes investigadores en sus estudios. Y si bien hay acuerdos en algunos de sus aspectos, "no es fácil hablar de autoevaluación de los aprendizajes en educación superior porque no se habla el mismo idioma, es decir, no hay un consenso sobre el concepto, existen ideas equivocadas" (LEACH, 2012, p. 138). ¿Qué ha impedido comprender la autoevaluación? ¿Se han considerado todos los aspectos que ella abarca?

Al analizar el concepto autoevaluación desde varios autores que han escrito sobre él en los últimos 30 años se evidencian posturas diversas. Así, encontramos la idea de proceso de reflexión, de autocrítica y análisis que desarrolla hábitos de reflexión, como sostiene Santos Guerra (1994). Por otro lado, Boud plantea que la autoevaluación tiene que ver con "la participación de los estudiantes en la identificación de los estándares y/o los criterios para aplicar en su trabajo, y juzgar en qué medida ellos han logrado estos estándares y criterios" (1991, p. 5, como se cita en BOUD, 1995, p. 12). Se comprende entonces que el "sí mismo", el estudiante, es valorado y tenido en cuenta como persona responsable, capaz de proponer puntos de referencia, que es la base para las "cualidades relativas" que explicaba Ortega y Gasset anteriormente, pues ellos -los puntos de referencia- provienen de los diferentes sujetos, y sin ellos no existe una valoración adecuada.

Para Boud (1995, p. 11), cuando se evalúa, se consideran dos elementos clave:

El primero es el desarrollo del conocimiento y una apreciación de los estándares apropiados y los criterios para satisfacer estos estándares, que podrían ser aplicados en cualquier trabajo. Si no se sabe qué cuenta como un buen trabajo, es imposible decir si un trabajo específico es adecuado. El segundo es la capacidad de hacer juicios sobre si el trabajo que se considera, satisface o no estos estándares. Desafortunadamente, el énfasis normalmente se hace, por ambos, profesores y estudiantes, al segundo de estos dos elementos.

Los aportes de Boud y Brew (1995) se relacionan con la diferencia y la similitud entre la reflexión y la autoevaluación. Para ellos, si bien la reflexión y la autoevaluación se conectan al aprendizaje y la experiencia, la autoevaluación involucra la reflexión para hacer juicios sobre logros; en cambio, la reflexión es una actividad más exploratoria que no siempre lleva a la autoevaluación.

Panadero Calderón (2011, p. 78) se refiere a la autoevaluación como una "valoración cualitativa de la ejecución y el producto final obtenido a partir de unos criterios de evaluación". Él manifiesta que hay “dos vertientes teóricas” sobre la autoevaluación. La primera, al no hacer énfasis en el proceso interno de la persona, es más “[...] como estrategia instruccional, se parte de una visión más pedagógica en línea con los trabajos sobre 'evaluación auténtica' - 'formative assessment'- (BLACK; WILLIAM, 1998, 2009)" (PANADERO CALDERÓN, 2011, p. 78). 
La segunda vertiente se sustenta en las teorías sobre autorregulación, y "es entendida como un subproceso que el alumno realiza para autorregular su aprendizaje [...], se centra en el proceso interno y no se suele explicitar como recurso instruccional". ¿Esas ideas no confirman los aspectos psicológico-sociológicos de la autoevaluación, con sus implicaciones pedagógicodidácticos?

De las posturas de los autores citados se comprende que la autoevaluación como un tipo de evaluación incluye los mismos momentos que esta, pero es la valoración interior del propio aprendizaje la que genera autoobservación y autorreflexión. De la misma manera, se devela claramente el rol del profesor para incluir la autoevaluación como una práctica pedagógica desde la enseñanza, pero en un espacio de comunicación abierta, de diálogo con el estudiante; y este, al pensar por sí mismo y al ser guiado por el profesor, realizaría la autoevaluación para regular su aprendizaje, para aprender a aprender -en el sentido de tener consciencia del proceso como tal- y para crecer como persona. Varios académicos han propuesto clasificaciones con base en diferentes criterios, en trabajos publicados entre 1995 y 2010. Estas se presentan de manera breve en la tabla 1 .

Tabla 1 - Clasificación de la autoevaluación

\begin{tabular}{|c|c|c|c|c|}
\hline Autores & \multicolumn{3}{|c|}{ Tipos de autoevaluación según los autores } & \multirow{2}{*}{$\begin{array}{c}\text { Criterios de la } \\
\text { clasificación }\end{array}$} \\
\hline $\begin{array}{l}\text { Boud } \\
\text { (1997) }\end{array}$ & $\begin{array}{l}\text { Opresiva: una vez, no } \\
\text { se prepara, cuantitativa, } \\
\text { otros. }\end{array}$ & & $\begin{array}{l}\text { Liberadora: sistemática, } \\
\text { cualitativa, otros. }\end{array}$ & \\
\hline $\begin{array}{l}\text { Tan } \\
(2009)\end{array}$ & $\begin{array}{l}\text { Teacher-driven: } \\
\text { (dirigido por el } \\
\text { profesor) } \\
\text { basado en la autoridad } \\
\text { del profesor. }\end{array}$ & $\begin{array}{l}\text { Programme-driven } \\
\text { (conducido por el } \\
\text { programa): } \\
\text { cumplimiento del } \\
\text { programa de estudio. }\end{array}$ & $\begin{array}{l}\text { Future-driven (impulsado } \\
\text { por el futuro): } \\
\text { desarrollo de capacidades. }\end{array}$ & $\begin{array}{l}\text { Concepciones } \\
\text { de profesores } \\
\text { sobre la } \\
\text { autoevaluación. }\end{array}$ \\
\hline $\begin{array}{l}\text { Boud y } \\
\text { Brew } \\
(\mathbf{1 9 9 5 )}\end{array}$ & $\begin{array}{l}\text { Interés técnico: } \\
\text { se comparan las } \\
\text { respuestas a partir de } \\
\text { un modelo con } \\
\text { criterios. }\end{array}$ & $\begin{array}{l}\text { Interés comunicativo: los } \\
\text { alumnos discuten qué es } \\
\text { una "buena" respuesta, } \\
\text { buscan o negocian los } \\
\text { criterios que aplican. }\end{array}$ & $\begin{array}{c}\text { Interés emancipatorio: } \\
\text { los alumnos discuten } \\
\text { criterios, hacen preguntas, } \\
\text { son críticos, } \\
\text { autorreflexionan. }\end{array}$ & $\begin{array}{c}\text { Teoría de } \\
\text { Habermas sobre } \\
\text { los intereses de } \\
\text { conocimiento } \\
\text { humano. }\end{array}$ \\
\hline $\begin{array}{l}\text { Taras } \\
(\mathbf{2 0 1 0})\end{array}$ & $\begin{array}{c}\text { Débil: } \\
\text { autocalificaciones y } \\
\text { similares ("sound } \\
\text { standards"). }\end{array}$ & $\begin{array}{c}\text { Mediano: } \\
\text { el modelo estándar. }\end{array}$ & $\begin{array}{c}\text { Fuerte: } \\
\text { autoevaluación con } \\
\text { retroalimentación (feedback) } \\
\text { del profesor/“learning } \\
\text { contract design”. }\end{array}$ & $\begin{array}{l}\text { Modelos de } \\
\text { autoevaluación, } \\
\text { según las } \\
\text { actividades } \\
\text { realizadas. }\end{array}$ \\
\hline $\begin{array}{l}\text { Andrade } \\
(2007- \\
2008)\end{array}$ & $\begin{array}{l}\text { Self-evaluation: } \\
\text { solo calificación. }\end{array}$ & & $\begin{array}{l}\text { Self-assessment: desarrollo } \\
\text { de capacidades (evaluar, } \\
\text { reflexionar, juzgar, buscar } \\
\text { caminos para mejorar). }\end{array}$ & $\begin{array}{l}\text { Intención, } \\
\text { propósito. }\end{array}$ \\
\hline
\end{tabular}

Fuente: elaboración propia. 
Podemos concluir que en las clasificaciones de la tabla 1, a pesar de algunas diferencias, se observan características comunes: la autoevaluación del aprendizaje depende del profesor y de su postura frente a la enseñanza, el aprendizaje y la evaluación. De ello interpretamos que puede ser cerrada o abierta, dependiente o independiente, relevante o insignificante, obediente o transformadora.

En otras palabras, si la autoevaluación se enfoca desde una mirada multidimensional, se comprende como un asunto complejo que trasciende la autocalificación que por lo general se solicita al estudiante al finalizar una asignatura a partir de criterios elaborados por los profesores. Los sentidos de la autoevaluación son mucho más profundos que los resultados obtenidos con una acción esporádica que se aplica para presumir que la autoevaluación se utiliza con los estudiantes, pero que en realidad no logra en ellos la formación a futuro y el desarrollo de capacidades para aprender a lo largo de la vida.

\section{Cuáles confusiones persisten sobre el concepto autoevaluación}

Una vez revisada la evolución del concepto de autoevaluación, descubrimos que aún persisten algunos malentendidos. Desde el inicio del surgimiento del término, al parecer siempre se relacionó con la calificación y la medición. Es de señalar que en inglés existen dos conceptos diferentes: self-evaluation y self-assessment, a menudo traducidos al español solo como autoevaluación. El primero es equivalente a la autoevaluación sumativa o autocalificación, donde evaluation significa "acción de evaluación" o "valoración”, del francés évaluer, "para encontrar el valor de" (ONLINE ETYMOLOGY DICTIONARY [s. f.]).; mientras que el segundo corresponde a la autoevaluación formativa o autovaloración, donde assessment, proveniente del latín assidere, significa estar sentado al lado de alguien (BIRENBAUM, 1996).

En este sentido, Taras (2015, p. 200) afirma que la autoevaluación ha cambiado en comparación con los años ochenta, dado que ahora se centra más "en desarrollar juicios autónomos que en la calificación". No obstante, si bien hay algún avance en las autoevaluaciones implementadas, este es muy pequeño, pues aún existe la tendencia de utilizar la self-evaluation frente a la self-assessment. Por ejemplo, así lo confirma Canney Villa (2014) en su investigación, en la que se evidenció una preponderancia de la autoevaluación como autocalificación; es decir, tanto el estudiante como el docente están más interesados en obtener una calificación que en reflexionar sobre el proceso, hecho al que la autora da una posible explicación: la poca importancia y la falta de interés que se le da al proceso de autoevaluación por parte del estudiante y de las instituciones educativas. 
Otros autores también señalan diversas razones para interpretar lo que sucede con la autoevaluación del aprendizaje en la educación superior. Así, para Andrade (2007-2008), la confusión entre self-evaluation y self-assessment (la diferencia es sutil, pero muy poderosa en la práctica) ha llevado a un mal entendido y a cierto rechazo de la autoevaluación. Los profesores dudan en utilizarla, al afirmar que los alumnos siempre se van a dar una calificación alta y que solo a veces van a revisar su trabajo, por lo cual no es necesario tomar tiempo para realizar la autoevaluación.

Por su parte, Bourke (2014) señala que la autoevaluación no es legítima en la universidad debido a tres factores: el fuerte poder de los profesores, el rol pasivo del estudiante y las políticas organizacionales que todavía no la institucionalizan. Trevitt, Breman y Stocks (2012) consideran que la autoevaluación se puede aplicar cuando el aprendizaje es concebido como una construcción. Cuando se parte de la convicción de que el aprendizaje se construye con la ayuda de los demás -profesores, compañeros u otros-, entonces cambia la visión de la evaluación, lo que lleva a utilizar la autoevaluación.

Además de estas restricciones en el uso de la autoevaluación, también se han develado otros significados. Falchikov (2005) señaló siete tipos de beneficios de la autoevaluación: mejora en las competencias cognitivas y metacognitivas; desarrollo de una variedad de habilidades; desempeño mejorado; desarrollo personal o intelectual, o de competencias sociales optimizadas; mejores "disposiciones afectivas"; mejores evaluaciones, y beneficios para los maestros.

Como desventajas de la autoevaluación se consideran la actitud negativa de los estudiantes, el temor que sienten frente a ella (SIOW, 2015) y la desconfianza en la honestidad de los estudiantes por parte de los profesores. No obstante, ¿será que dichas desventajas están presentes y se atribuyen a la autoevaluación porque ha faltado comprenderla desde todos sus significados ya develados en el concepto? ¿Será que en el ámbito académico de la educación superior se necesita más reflexión, discusión, conocimiento, investigación, formación, compromiso y consciencia sobre la autoevaluación del aprendizaje, antes de rechazarla, criticarla, aislarla o utilizarla a medias?

El estudiante universitario debe tener derecho de "intervenir en su evaluación", porque ella porta valor educativo y formativo, y es "el vínculo entre el trabajo del grupo y la responsabilidad individual [...], del egresado, como exigencia a la Educación Superior. Se establece como propósito legítimo que el evaluado devenga evaluador" (GONZÁLEZ PÉREZ, 2000, p. 11). 
Así mismo, la autoevaluación es un asunto ontológico; es decir, tiene que ver con el ser, a quien le interesa el conocimiento no como un fin, sino como parte del camino para convertirse en un profesional; un ser que posee dicho conocimiento y valora el significado de aprender y ser capaz de aplicar lo aprendido en su práctica y en su vida cotidiana. Se trata no solo de lo que sabemos, sino también de quiénes somos y cómo actuamos (BOURKE, 2017).

La autoevaluación, además, podría desarrollar la capacidad de ser evaluadores del aprendizaje, "una habilidad típicamente omitida de la lista de habilidades claves para los graduados, pero implícita en la noción de aprender a aprender y en llegar a ser aprendiz a lo largo de la vida" (BOUD; FALCHIKOV, 2006, p. 402). Al evaluar a los estudiantes todo el tiempo sin estimularlos a autoevaluarse, ellos siempre reciben otros juicios, pero no exploran los propios; por lo tanto, "es posible que no adquieran el conjunto más amplio de habilidades que les permita hacer esto por sí mismos" (BOUD; FALCHIKOV, 2006, p. 403).

Para Gadamer (1998, p. 62): "tener la capacidad de juicio es ser capaz de subsumir y aplicar correctamente lo que se ha aprendido y lo que se sabe [...], subsumir algo particular bajo una generalidad, reconocer algo de una regla.". La capacidad de juicio "no puede enseñarse en general sino sólo ejercerse una y otra vez [...]" (p. 63). Para que ello sea posible, los profesores deben desarrollar actividades de autoevaluación en el aula a diario, en todas las asignaturas, durante todas las carreras.

Para Boud y Brew (1995), la autoevaluación puede ser utilizada como un proceso o como una actividad, y también es una práctica y una meta a la vez, una meta en la educación superior. Díaz-Barriga (2006) coincide con los autores anteriores en que la autoevaluación con las habilidades y actitudes que requiere para su ejercicio debe ser "objeto de una enseñanza explícita" (DÍAZ-BARRIGA, 2006, p. 157). Sin embargo, ella llama la atención sobre un asunto muy importante:

[...] no basta con pedir al alumno que exprese abiertamente qué opina de su crecimiento en el curso o sobre los aprendizajes puntuales logrados, tiene que contarse con evidencia explícita y argumentos razonados. Es un error pensar que por la carga interpretativa, la apertura a la subjetividad o el carácter cualitativo del juicio experto que se tiene que emitir se puede dejar de lado un manejo riguroso sistemático y transparente de la información que se toma como base para emitir dicho juicio.

Así, el desarrollo de la capacidad de juicio de valor en la autoevaluación se basa en elementos lógicos, en algún tipo de pruebas, y no es puramente opinión sin fundamentos, lo que permite potenciar el pensamiento y el razonamiento en cada estudiante. 


\section{Conclusiones y reflexiones}

Al aproximarse a la historia del concepto autoevaluación, se comprende entonces que en la autoevaluación confluyen aspectos psicológico-sociológicos del self, filosóficoontológicos del ser y pedagógico-didácticos del sujeto que se forma para sí mismo y para convivir en la sociedad. Dicho sujeto, como un ser irrepetible con cognición y emoción, saber y acción, actúa desde sí hacia los demás; y cuando es evaluador y evaluado, a partir de sus criterios discutidos y consensuados con los de otros, desarrolla juicios de valor, además de potenciar capacidades que se transfieren en diferentes situaciones y facilitan el aprendizaje a lo largo de la vida. La autoevaluación y la evaluación se complementan en un proceso evaluativo (como unidad) justo y democrático. La primera no es una opción, es parte del todo.

Entonces, como síntesis (la cosa creada), la autoevaluación del aprendizaje es un proceso individual de conocimiento de sí mismo en relación con lo académico, desde la libertad y la autodeterminación, en una interacción con el otro (el profesor), quien reconoce al estudiante como un ser humano y lo respeta, al igual que sus posturas; por lo tanto, cree en él y le brinda confianza para aprender desde los propósitos formativos, los contenidos generales y la evaluación, pero considerando sus necesidades e intereses particulares para desarrollar capacidades, habilidades y destrezas que lo guíen en su aprendizaje hacia el futuro, con el fin de realizar su proyecto de vida e incidir en la transformación de la sociedad.

Sin embargo, el concepto autoevaluación aún no logra ser comprendido desde dichas perspectivas en la comunidad académica, y no permea las prácticas de los profesores en el proceso de enseñanza-aprendizaje en educación superior. Las secuelas del pasado dejan su sombra al frenar el abordaje de la autoevaluación en el tiempo actual. Entre ellas están: la fuerte tradición de la evaluación sumativa; la importancia de lo cuantitativo como máxima expresión del rigor científico y la objetividad heredados del positivismo (que ha marcado hasta la misma autoevaluación, pues ella también es sumativa y formativa); la visión del estudiante como objeto y no como sujeto en el proceso de enseñanza-aprendizaje, una influencia del conductismo y la óptica de un ser fragmentado alejada de la tendencia humanista; la presencia de posturas sobre el aprendizaje como transmisión y acumulación de conocimiento, contrario a la construcción individual, que incluye las esferas de lo cognitivo, lo emocional y lo social; la predominancia de profesores con "competencia disciplinar (como conocedores fidedignos del ámbito científico que enseñan)" sobre la "competencia pedagógica (como personas comprometidas con la formación y el aprendizaje de sus estudiantes)" (ZABALZA BERAZA, 2011, p. 413) en las instituciones educativas de nivel superior. 
Mientras no se avance hacia nuevos horizontes en la comprensión de la autoevaluación del aprendizaje y no se emprendan acciones para superar las limitaciones mencionadas, esta seguirá siendo un hecho aislado y voluntario, supeditada al deseo, la convicción, el tiempo y la oportunidad del profesor, en lugar de ser un componente indispensable dentro del proceso de enseñanza-aprendizaje. Al considerarse como tal, trasciende a los espacios universitarios netamente disciplinares, dado que apuesta a la formación del ser humano; en ese espacio, el rol del profesor no es solo de cuidar de sí mismo, sino ocuparse "del cuidado que pueda tener sobre sí mismo aquel a quien él sirve de guía" (FOUCAULT, 1987, p. 49).

\section{Referencias}

ÁLVAREZ ROJO, Víctor et al. Análisis de la participación del alumnado universitario en la evaluación de su aprendizaje. Revista Española de Pedagogía, Madrid, v. 69, n. 250, p. 401426, sep./dic., 2011.

ANDRADE, Heidi. Self-assessment through rubrics. Educational Leadership, Virginia, Estados Unidos, v. 65, n. 4, p. 60-63, Dec./Jan., 2007-2008. Disponible en: https://imoberg.com/files/2008_Self-Assessment_through_Rubrics.pdf. Acceso en: 23 abr. 2019.

ASHMORE, Richard; JUSSIM, Lee (ed.). Self and Identity. Fundamental Issues. Nueva York: Oxford University Press, 1997.

BIRENBAUM, Menucha. Assessment 2000: towards a pluralistic approach to assessment. In: BIRENBAUM, Menucha; DOCHY, Filip (eds.). Alternatives in assessment of achievements, learning processes and prior knowledge. Nueva York: Kluwer Academic Publishers, 1996. Disponible en:

https://books.google.com.co/books?id=d661BwAAQBAJ\&printsec=\%20frontcover\&source= gbs_ge_summary_r\&cad=0\#v=onepage\&q\&f=false. Acceso en: 23 abr. 2019.

BOUD, David. Enhancing learning through self-assessment. Londres; Nueva York: Routledge Falmer; Taylor \& Francis Group, 1995.

BOUD, David. The Move to Self-Assessment: Liberation or a New Mechanism for Oppression? Reproduced from 1994 Conference proceedings, Scutrea, p. 10-13, 1997. Disponible en: http://www.leeds.ac.uk/educol/documents/00002954.htm. Acceso en: 23 abr. 2019.

BOUD, David; BREW, Angela. Developing a typology for learner self-assessment practices. Research and Development in Higher Education, Sídney, n. 18, p. 130-135, 1995. Disponible en: https://www.researchgate.net/publication/327350493_Developing_a_typology_for_learner_se lf-assessment_practices. Acceso en: 23 abr. 2019. 
BOUD, David; FALCHIKOV, Nancy. Aligning assessment with long-term learning. Assessment and EVALUATION IN HIGHER EDUCATION, Londres, v. 31, n. 4, p. 399413, 2006. Disponible en: http://www.jhsph.edu/departments/population-family-andreproductive-health/_docs/teaching-resources/cla-01-aligning-assessment-with-long-termlearning.pdf. Acceso en: 22 abr. 2019.

BOURKE, Roseanna. Self-assessment in professional programmes within tertiary institutions. Teaching in Higher Education, Abingdon-on-Thames, Reino Unido v. 19, n. 8, p. 908-918, 2014. Disponible en: http://dx.doi.org/10.1080/13562517.2014.934353. Acceso en: 22 abr. 2019.

BOURKE, Roseanna. Self-assessment to incite learning in higher education: developing ontological awareness. Assessment and Evaluation in Higher Education, Londres, v. 43, n. 5, p. 827-839, 2017. Disponible en: https://www.tandfonline.com/doi/abs/10.1080/02602938.2017.1411881. Acceso en: 22 abr. 2019.

BREW, Angela. What is the scope of self-assessment? In: BOUD, David. Enhancing Learning Through Self-Assessment. Londres; Nueva York: Routledge Falmer; Taylor \& Francis, 1995. p. 48-62.

CABRALES SALAZAR, Omar. Evaluación de los aprendizajes en estudios de posgrado. Educación y Desarrollo Social, Bogotá, v. 4, n. 1, p. 10-37, 2010.

CANNEY VILLA, María Patricia. La autoevaluación por el estudiante: aproximación a las creencias de docentes universitarios. 2014. 64 p. Tesis (Maestría) - Pontificia Universidad Javeriana, Bogotá D. C., 2014. Disponible en:

https://repository.javeriana.edu.co/handle/10554/12356. Acceso en: 22 abr. 2019.

CANTO-SPERBER, Monique (dir.). Diccionario de ética y de filosofía moral (K-W). México: Fondo de Cultura Económica, 2001. Tomo II.

CLONINGER, Susan. Teorías de la personalidad. 3. ed. México: Pearson Educación, 2003.

COROMINAS, Joan. Breve diccionario etimológico de la lengua castellana. 3. ed. Madrid: Gredos, 1987.

DECLARACIÓN DE BOLONIA. 1999. Disponible en:

http://eees.umh.es/contenidos/Documentos/DeclaracionBolonia.pdf. Acceso en: 23 abr. 2019.

DÍAZ-BARRIGA, Frida. Enseñanza situada: vínculo entre la escuela y la vida. México: McGraw-Hill, 2006.

FALCHIKOV, Nancy. Improving assessment through student involvement: practical solutions for aiding learning in higher and further education. Londres: Routledge Falmer, 2005 .

FOUCAULT, Michel. Hermenéutica del sujeto. Madrid: Ediciones La Piqueta, 1987. 
FOUCAULT, Michel Tecnologías del yo y otros textos afines. 2. ed. Barcelona: Ediciones Paidós Ibérica, 1991.

FRAGER, Robert; FADIMAN, James. Carl Rogers y la perspectiva centrada en la persona. In: FADIMAN, James; FRAGER, Robert. Teorías de la personalidad. 2. ed. Bogotá: Alfaomega; Oxford: Oxford University Press, 2001. p. 312-455.

GADAMER, Hans-Georg. Verdad y método II. Salamanca: Ediciones Sígueme, 1998.

GAIRÍN SALLÁN, Joaquín. La autoevaluación institucional como vía para mejorar los centros educativos. Revista Educación y Ciencias Humanas, Caracas, v. 5, n. 8, p. 34-67, 1997.

GONZÁLEZ AGUDELO, Elvia María. Sobre la experiencia hermenéutica o acerca de otra posibilidad para la construción del conocimiento. Discusiones Filosóficas, Manizales, v. 12, n. 18, p. 125-143, 2011.

GONZÁLEZ PÉREZ, Miriam. Evaluación del aprendizaje en la enseñanza universitaria. Pedagogía Universitaria, La Habana, v. 5, n. 2, 2000. Disponible en: https://profesorailianartiles.files.wordpress.com/2013/04/evaluacic3b3n-del-aprendizaje-enensec3b1anza-universitaria-parte-1.pdf. Acceso en: 24 abr. 2019.

HERON, John. Assessment revisited. In: BOUD, David (ed.). Developing student autonomy in learning. Londres: Kogan Page, 1988. Disponible en: http://www.humaninquiry.com/asre.htm. Acceso en: 22 abr. 2019.

HORLACHER, Rebekka. ¿Qué es Bildung? El eterno atractivo de un concepto difuso en la teoría de la educación alemana. Pensamiento Educativo: Revista de Investigación Educacional Latinoamericana, Santiago de Chile, v. 51, n. 1, p. 35-45, 2014. Disponible en: https://pensamientoeducativo.uc.cl/index.php/pel/article/view/617. Acceso en: 22 abr. 2019.

JESÚS, Girlene Ribeiro de; BEDRITICHUK, Amanda Guedes Andrade. Autoavaliação institucional: construção e validação de um questionário para o professor. Avaliação, Campinas; Sorocaba, SP, v. 23, n. 3, p. 628-647, nov. 2018. Disponible en: https://www.scielo.br/scielo.php?script=sci_abstract\&pid=S141440772018000300628\&lng=en\&nrm=iso\&tlng=pt. Acceso en: 22 abr. 2019.

LANZ, César. El cuidado de sí y del otro en lo educativo. Utopía y Praxis Latinoamericana: Revista Internacional de Filosofía Iberoamericana y Teoría Social, Maracaibo, v. 17, n. 56, p. 39-46, 2012.

LASONEN, Johanna. A case study of student self-assessment in upper secondary education. In: Contemporary Issues of Occupational Education in Finland, Jyvaskyla, p. 119-215, 1995. Disponible en: https://files.eric.ed.gov/fulltext/ED386561.pdf. Acceso en: 24 abr. 2019.

LEACH, Linda. Optional self-assessment: some tensions and dilemmas. Assessment and Evaluation in Higher Education, Londres, v. 37, n. 2, p. 137-147, 2012. Disponible en: https://eric.ed.gov/?id=EJ954991. Acceso en: 22 abr. 2019. 
MACARIO OCAMPOS, Gustavo. La filosofía socrática y el autoconocimiento; una búsqueda continua de sí mismo. 2017. Trabajo de grado (Licenciatura en Filosofía) Universidad Autónoma del Estado de México, Toluca, 2017.

MASLOW, Abraham. Some educational implications of the humanistic psychologies. Harvard Educational Review, Cambridge, v. 38, n. 4, p. 685-696, 1968. Disponible en: http://ocw.metu.edu.tr/pluginfile.php/9045/mod_resource/content/1/fulltext.pdf. Acceso en: 24 abr. 2019.

MINISTERIO DE EDUCACIÓN NACIONAL. El sistema de educación superior de Colombia. 2007. Disponible en: https://www.cna.gov.co/1741/article-187279.html. Acceso en: 17 abr. 2019.

ONLINE ETYMOLOGY DICTIONARY. Disponible en: https://www.etymonline.com/search?q=evaluation. Acceso en: 24 abr. 2019.

ORTEGA Y GASSET, José. Introducción a una estimativa. ¿Qué son los valores? Madrid: Encuentro Ediciones, 2004. Disponible en: https://www.salud.gob.ec/wpcontent/uploads/2016/12/2.2.-Ortega-Que-son-los-valores.pdf. Acceso en: 24 abr. 2019.

PANADERO CALDERÓN, Pedro Ernesto. Ayudas instruccionales a la autoevaluación y la autorregulación: evaluación de la eficacia de los guiones de autoevaluación frente a la de las rúbricas. 2011. Tesis (Doctorado) - Universidad Autónoma de Madrid, 2011. Disponible en: https://repositorio.uam.es/handle/10486/10832. Acceso en: 17 abr. 2019.

REAL ACADEMIA ESPAÑOLA. Diccionario de la Real Academia Española. 2001. Disponible en: http://dle.rae.es/?id=BRNxtDx. Acceso en: 22 abr. 2019.

RIVEROS AEDO, Edgardo. La psicología humanista: sus orígenes y su significado en el mundo de la psicoterapia a medio siglo de existencia. Ajayu, La Paz, v. 12, n. 2, p. 135-186, 2014. Disponible en: http://www.scielo.org.bo/pdf/rap/v12n2/v12n2a1.pdf. Acceso en: 22 abr. 2019.

ROGERS, Carl. A theory of therapy, personality, and interpersonal relationships, as developed in the client-centered framework. 1959. Disponible en: https://www.academia.edu/29021825/A_THEORY_OF_THERAPY_PERSONALITY_AND INTERPERSONAL_RELATIONSHIPS_AS_DEVELOPED_IN_THE_CLIENTCENTERED_FRAMEWORK. Acceso en: 18 mayo 2019.

SANTOS GUERRA, Miguel Ángel. Entre bastidores. Malaga: Aljibe, 1994.

SIOW, Lee-Fong. Students' perceptions on self- and peer- assessment in enhancing learning experience. The Malaysian Online Journal of Educational Science, Kuala Lumpur, v. 3, n. 2, p. 21-35, 2015. Disponible en: http://files.eric.ed.gov/fulltext/EJ1085908.pdf. Acceso en: 20 abr. 2019.

TAN, Kelvin. Meanings and practices of power in academics' conceptions of student selfassessment. Teaching in Higher Education, Reino Unido, v. 14, n. 4, p. 361-373, 2009. Disponible en: https://www.tandfonline.com/doi/abs/10.1080/13562510903050111. Acceso en: 20 abr. 2019. 
TARAS, Maddalena. Student self-assessment: processes and consequences. Teaching in Higher Education, Reino Unido, v. 15, n. 2, p. 199-209, 2010.

TARAS, Maddalena. Autoevaluación del estudiante: ¿Qué hemos aprendido y cuáles son los desafíos? Relieve, Valencia, v. 21, n. 1, 2015. Disponible en:

https://ojs.uv.es/index.php/RELIEVE/article/view/6394. Acceso en: 24 abr. 2019.

TREVITT, Chris; BREMAN, Elinor; STOCKS, Claire. Assessment and learning: is it time to rethink student activities and academic roles? Revista de Investigación Educativa,

Murcia, v. 30, n. 2, p. 253-267, 2012. Disponible en:

http://revistas.um.es/rie/article/viewFile/153441/1397 HYPERLINK

"http://revistas.um.es/rie/article/viewFile/153441/139701"01. Acceso en: 24 abr. 2019.

TRONCHIN, Daisy Rizatto; PEDRO, Aisha Negreiros da Costa; REZENDE, Daniele Pereira. Métodos avaliativos da aprendizagem no bacharelado na Escola de Enfermagem da Universidade de São Paulo. Avaliação, Campinas; Sorocaba, SP, v. 22, n. 3, p. 758-771, nov. 2017. Disponible en: http://www.scielo.br/pdf/aval/v22n3/1982-5765-aval-22-03-00758.pdf. Acceso en: 24 abr. 2019.

WEBSTER, Murray; SOBIESZEK, Barbara. Teorías de la autoevaluación. Estudio experimental de psicología social. México: Limusa, 1978.

ZABALZA BERAZA, Miguel Ángel. Nuevos enfoques para la didáctica universitaria actual. Perspectiva, Florianópolis, v. 29, n. 2, p. 387-416, jul./dic., 2011. 